\title{
Simulation: Pre-briefing Preparation, Clinical Judgment and Reflection. What is the Connection?
}

\section{Simulasyon: Brifing Öncesi Hazırlık, Klinik Yargı ve Yansıma. İlişki Nedir?}

\author{
Leighsa Sharoff ${ }^{1}$
}

\begin{abstract}
${ }^{1}$ School of Nursing, Associate Professor City University of New York at Hunter
\end{abstract}

\section{Corresponding Author:}

Dr. LeighsaSharoff

\section{Address:}

School of Nursing, Associate Professor City University of New York at Hunter

Fax: 212.481 .4427

E-mail:

1sharoff@hunter.cuny.edu

Başvuru Tarihi/Received : 09-05-2014

Kabul Tarihi/Accepted: 16-09-2014

\section{ABASTRACT}

Objectives: Assess efficacy of pre-briefing preparatory materials handed out prior to simulation lab and explore the connection between simulation, clinical judgment and reflective process. Methods:Triangulated evaluation study, utilizing Survey Monkey for three survey instrument data collection, designed to assess the efficacy of preparatory materials for undergraduate junior and senior nursing students and nurse educators while exploring the connection with clinical judgment and reflection. This study was conducted in the U.S.A.

Results:Pre-briefing preparatory material for both students and instructors provided essential and adequate information for an effective and enhanced simulation learning experience. Students felt their ability to grasp the experience; understand the simulation situation; provide effective nursing actions; and reflect upon their experience was enhanced with the utilization of the pre-briefing preparatory material. Instructors felt more confident and prepared after reading the preparatory material.

Conclusions:Pre-briefing preparatory material provides students with information necessary to allow them to fully engage during the simulation experience, while decreasing anxiety and promoting critical thinking, clinical reasoning, reflective practice and confidence. Providing instructors with pre-briefing preparatory material enhances their overall ability to effectively teach at a higher caliber while promoting professionalism and accuracy in patient care.

Key Words: Simulation, pre-briefing preparation, critical thinking, reflective practice, clinical judgment, education 


\section{INTRODUCTION}

High-fidelity human patient simulation (HFS) provides an environment that as realistically as possible reflects a clinical setting, offering students an opportunity to enhance diverse learning needs in a safe environment. Opportunities to improve assessment skills, gain insight into the decision making process and the consequences of those decisions can occur with simulation [1].

Several studies have explored simulation [2-4].There have been studies exploring how well simulation prepares students for clinical judgment development[2,5,6] yet studies are still needed to explorethe reflective process that is intertwined with clinical reasoning to further enhance knowledge retention. Clinical judgment is quite complex, and necessitates a flexible and distinct ability to recognize undefined clinical information, interpret its meaning and respond appropriately [7,8].In conjunction with clinical judgment is reflection on practice which is critical for the development of clinical knowledge and improvement in clinical reasoning [5,7].Reflecting upon one's experience promotes learning, and a guide for reflection using the clinical judgment model was developed to promote students' reflective writing to encourage critical thinking, knowledge transfer, transformation, metacognition, and exploration of emotional aspects of situations encountered in clinical experiences [9,10]. Clinical judgment and reflection are essential to the development of future health care providers.

While simulation is an important component of clinical training,educators need to learn the most effective and efficient way to prepare faculty and students for the experience while promoting reflective practice to ensure appropriate clinical judgment. Numerous studies have focused on student and faculty perceptions [3,11-12]. An area that necessitates further research is how best to prepare both faculty and students for the simulation experience.With health care reform such a prominent issue, nursing schools will be required to train more students with limited clinical resources, making simulation an increasingly important and effective strategy. To effectively educate the $21^{\text {st }}$ century nursing student, it is imperative that nursing curricula stay abreast of current mandated competencies. The design of pre-briefing preparatory materials must enhance students' knowledge base, skills acquisition, required competencies and learning outcomes [13]. Learning outcomes, skill performance, critical thinking, self-confidence, and communicative abilities all constitute the construct of simulation success in enhancing students' abilities [14,15].

Strategies were devised to implement simulation in nursing curricula [16,17]. Understanding the role of pre-briefing preparation, clinical judgment and reflective practice is now warranted to further explore how best to integrate simulation into all realms of health care. Consequently, is there an enhancement of students' clinical judgment and reflection? Does providing pre-briefing preparatory material in advance of the simulation experience enhance students' learning outcomes and simulation objectives? Does preparatory material assist educators in providing a better understanding of nursing actions during the simulation?

This study explored the efficacy of simulation pre-briefing preparatory material that is currently being utilized by faculty and students in a diverse urban undergraduate School of Nursing (SON) program. It also explored the connection of simulation to clinical judgment and reflection.As the Simulation Coordinator for a large urban SON in the USA, the researcher developed student and faculty preparatory templates, based on NLN case study simulation scenarios, including all the 


\section{DOI: $10.16899 /$ ctd.49922}

advised strategies as well as additional innovative, simulating and engaging strategies, incorporating YouTube videos, educational CDs from the University Health Professions Educational Center; and extensive information pertaining to the scenario medical condition (including physiology, pathophysiology, complications and images) to prepare students and educators. Pre-briefing preparatory materials are handouts that provide an overview of the simulation case scenario. Suggested information can include a point of reference to equipment in use during the simulation, the simulated patient environment and manikin, student roles during the simulation, time allotment, simulation objectives and patient situation (The INASCL Board of Directors, 2011).

See Table 1 for a Sample of Pre-briefing Preparatory Material

Table 1: Sample of Pre-briefing Preparatory Material

All participants received this pre-briefing preparatory material. Instructors' preparatory material has additional information.

Simulation Information: Cerebral Vascular Accident (CVA) - Ischemic Stroke

Mrs. Willamena Edwards

Simulation Information: Please acknowledge that the simulation experience is fiction. It is obviously 'not real' but as close to a real-life experience that we can offer. Be fair about simulator strengths and weaknesses. Given the simulator's limitations, we'll do our best to help make the simulation seem as real as possible; you have to do your part and act as if everything is real. That's how we'll get the most value out of this experience. We know that you will probably conduct yourself differently in the simulation than in clinical, and that's OK because there is still a lot to learn and talk about during this simulated experience.

You will be working in groups of 3-4 students. You will be expected to work as a team and function as the staff nurse. You will be expected to perform various assessments, identify treatment needs, provide patient education and engage in effective communication.

The entire scenario should take approximately 15-20 minutes. After the scenario, there will be a group debriefing, whereby the instructor will facilitate the group and discuss the various elements of nursing care (30-40 minutes).

\section{Roles during Simulation -}

As the students will be play-acting, each person in the group will be given a role for that simulation. The roles are:

- 2 primary care nurses. These individuals will provide for the majority of bedside care.

- Med RN - this individual will be the medication nurse during the scenario.

- Chart RN/Note Taker - this individual will be required to document all that occurs during the scenario in Scribe. You are responsible for viewing the simulation and taking notes on what was done/not done during the experience. This person provides a 'third-person' view of the simulation experience and adds to the rich discussion during the debriefing. Please be aware that this individual MUST report off/sign off at the end of the scenario, either via telephone or in person to Nurse Case Manager.

Scribe is our web-based Charting/Recording process. We will have laptops that have this downloaded on them for our use.

Please be prepared for this scenario and please do not worry too much. This is a practice exercise so you will be better prepared for 'real life' experiences in the hospital.

Prior to coming to the simulation, please make certain that you view the instructional videos on You-Tube written in your Simulation Overview. In addition, please view these:

Causes of a Stroke 3D Medical Show: https://www.youtube.com/watch?v=D2vPfOsTFbM

What is a Stroke? (Part 1 of 2)|HealthiNation: https://www.youtube.com/watch?v=nCqhnTedZMs

Stroke - Rehabilitation:

https://www.youtube.com/watch?v=9aaR542y-w8

Stroke: https://www.youtube.com/watch?v=LAzRf06BFsg

Secrets to Learn the NIH Stroke Scale:

https://www.youtube.com/watch?v=vIaLhIIHhS4

Go to this PDF for the NIH Stroke Scale: http://www.ninds.nih.gov/doctors/NIH_Stroke_Scale.pdf

Additional Resources:

A Tour of the Brain:

http://www.strokeassociation.org/STROKEORG/AboutStroke/E ffectsofStroke/ATouroftheBrain/A-Tour-of-the-

Brain_UCM_310943_Article.jsp

American Heart Association: Watch, Learn and Live, Interactive Cardiovascular Library \{Great visual explanations \}: http://watchlearnlive.heart.org/CVML_Player.php?moduleSelec $\underline{\mathrm{t}=\text { carsur }}$

\section{SIMULATION SCENARIO}

Willamena Edwards is a 65 year old female who presented to the emergency department at 10 am after waking up with right-sided weakness at 7:00am. Upon arrival, she was paretic on the right side with strength in upper and lower extremity rated as 3 out of 5 and she had right-sided facial weakness and garbled speech. Mrs. Edwards was evaluated as 7 on the NIH stroke scale. She has a peripheral IV running with $0.9 \%$ sodium 
chloride infusing at $75 \mathrm{~m} / \mathrm{L} /$ hour. Her CT scan revealed a non-

hemorrhagic stroke. Immunizations are current; annual flu shot. You are taking over the care of Mrs. Edwards.

Time: 10:30 am

\section{Clinical signs immediately visible:}

Alert and responsive

Patient's speech a little garbled

Has a drift of right-upper extremity

Patient data: Female, 65 age years old. Weight: 205 lbs

(93kg). Height: 73 inches (1.85 meters)

DOB: $05 / 18 / \mathrm{XX}$

MR\#: PCS51800

Religion: Christian

Allergies: Codeine (rash)

Major Support: Husband and daughter

Social History: Mrs. Edwards is a newly retired police

officer and she enjoys boating with her family.

Prior medical history: Mrs. Edwards has been diagnosed and treated by her own physician over the past years for high blood pressure, coronary heart disease and non-compliant diabetes (NIDDM). She still smokes 1 pack of cigarettes per day even though she has been told to quit. She is left-handed.

Recent medical history: Over the last couple of days, Mrs. Edwards has felt more uncomfortable than usual. Her husband has been very concerned about her but she refuses to see a doctor.

\section{Simulation Learning Objectives:}

Every simulation will have specific learning objectives. Learning objectives are building on your knowledge base. All students should be aware that the Comprehensive Learning Objectives are standards of care and build towards the Application Learning Objectives which further enhance your knowledge with the Synthesis Learning Objectives. The Comprehensive Learning Objectives are written in the Simulation Overview handout. The Application and Synthesis Learning Objectives are written in this handout. Please make certain you review ALL the objectives carefully.

Simulation Application Learning Objectives:

* Identifies the primary nursing diagnosis

* Implements patient safety measures

* Evaluates patient assessment information including vital signs

* Implements therapeutic communication

* Implements direct communication with multidisciplinary team members

* Demonstrates effective teamwork

* Prioritizes and implements Physician/Nurse Practitioner Orders appropriately

* Utilizes informatics in the coordination of patient care

* Maintains quality of care sickle cell anemia/sickle cell disease

* Utilizes findings from current research in the provision of client care

Simulation Synthesis Learning Objectives:

* Implements a focused cardiac and respiratory assessment

* Explains pain management in a patient with sickle cell anemia
* Initiates relevant cardiac and respiratory monitoring as needed

* Creates the primary nursing diagnosis

* Recalls \& implements policies related to legal

informed consent $\&$ information security

* Identifies the use of current medications if any, and allergy to medications

* Recalls indications, contraindications, and potential adverse effects of prescribed medications

* Implements the '7 rights' of medication administration

* Observes for desired effects, adverse effects of medications post administration

* Demonstrates appropriate nursing interventions in a patient with a CVA

* Identifies if patient is a candidate for thrombolytic therapy

* Implements correct stroke protocol

Pending Diagnostic Studies

Fasting lipid profile

$\mathrm{Hgb} \mathrm{A} 1 \mathrm{C}$ in am

Complete Medical Panel (CMP)

Complete blood count (CBC)

CT Scan

STAT Orders:

Keep systolic blood pressure between 140 and 180

If systolic blood pressure $>180$ give $5 \mathrm{mg}$ labetalol IV push over 1-2 minutes; may repeat times one

Call PCP if blood pressure is not controlled

Standing orders:

Daily CBC with differential and CMP

NPO/No Oral Intake

Suction PRN

Monitor I\&O every shift

Vital signs every 15 minutes for 2 hours; every 30 minutes times 6 hours; every 1 hour times 16 hours

Neuro checks every 15 minutes for 2 hours; every 30

minutes times 6 hours; every 1 hour times 16 hours

$0.9 \%$ sodium chloride infusing at $75 \mathrm{~mL} /$ hour

O2 saturation every 6 hours

$2 \mathrm{~L} / \mathrm{min} /$ wean to keep $\mathrm{SpO} 2>94 \%$

Blood sugar levels every 3 hours

Compression stockings

Bedrest for first 24 hours then up with assist

No lifting or pulling on plegic or paretic side

Assisted ADL

Full code status

Medications brought from home:

Metformin (Glucophage) 500mg PO BID

Glipizide (Glucotrol) 5mg PO daily

Hydrochlorathiazide 25mg PO daily

Lisinopril (Prinivil) 10mg PO daily

Simvastatin (Zocor) 40mg PO at bedtime

ASA PR 300 mg daily

Enoxaparin (Lovenox) 40mg subcutaneous daily

Please remember, the simulation experience can be very stressful. It is important to remind you that this is a learning experience which is not graded (except for the assignments). It is an opportunity for you to gain a deeper understanding of your actions and behavior. 


\section{THEORETICAL FRAMEWORK}

Literature on utilization, integration and implementation of simulation as a teaching strategy in nursing provided the study's framework. Tanner's model, derived from a synthesis of the literature on clinical judgment, is a rubric that defines stages or levels of clinical judgment development [7]. Simulation scenarios are leveled through nursing curricula to ensure a logical progression of skill and knowledge development. Students are exposed to increasingly complex simulation scenarios in order to progressively develop clinical judgment and clinical reasoning. Tanner's Clinical Judgment Model analyzed and described the complex model of clinical judgment, defining clinical judgment as "an interpretation or conclusion about a patient's needs, concerns or health problems, and/or the decision to take action (or not), to use or modify standard approaches, or to improve ones' as deemed appropriate by the patient's response" [7, p. 204]. The four phases to this model (Noticing, Interpreting, Responding, and Reflecting) correlate to clinical judgment as it relates to the multidimensional complexity of patient care.

An extensive review of reliable instruments to effectively explore clinical judgment yielded Lasater's Clinical Judgment Rubric (LCJR) and Scoring Sheet, which describes students' responses to simulated scenarios within the framework of Tanner's Clinical Judgment Model. The rubric offers language that is understood by both faculty and students, is used for student self-assessment of clinical judgment development through simulation and provides sets standards that students can comprehend and work toward $[18,19]$. The researchers' SON adapted Tanner's Clinical Judgment Model as its simulation conceptual framework as well as the LCJR.

To explore reflective practice and keep consistency with the conceptual framework, the researcher integrated the Guide for Reflection using Tanner's Clinical Judgment Model [9]. This guide, intended to help students think about a clinical situation, was adapted for the simulation situation and used to promote reflective practice. Reflective practice is the process of reflecting upon one's experience to make sense of that experience; enhance his/her knowledge base, and thus expands on clinical judgment and self-confidence which will contribute to improved patient care and outcomes [2, 5, 20-21].

\section{METHODS}

Participants were undergraduate baccalaureate students and clinical instructors; therefore, minimization of perceived 'coercion' was at the forefront. Confidentiality and anonymity was maintained via the utilization of Survey Monkey as the sole form of data collection, employing numerical numbers rather than names. Ethical consideration for all participants was at the forefront of this study. The researcher did not engage in any discussion on this study with any student enrolled in the Hunter College undergraduate baccalaureateSchool of Nursing program, thus eliminating coercion. The researcher did not have any administrative responsibilities regarding the instructors, eliminating coercion possibilities there as well. A research assistant (RA), a doctoral student from the City University of New York (CUNY) Graduate Center, was trained on study procedures and confidentiality and had no educational relationship with participants. She met the junior and senior classes, separately, at their scheduled didactic course to explain the study's purpose. The RA was responsible for emailing 
the surveys to all participants and answering any inquiries on this study. IRB approval was obtained. A \$5.00 donation to the American Cancer Society was made for completed surveys. There was no conflict of interest with the researcher and the American Cancer Society. Study was conducted during 20102011; consent forms obtained for all participants.

Participants: All undergraduate junior and senior nursing students were invited to participate as were their clinical/simulation instructors. A total of 81 students and 9 instructors participated. Overall, students' age ranged from 19-52 years old (mean 21.8 years). Seventy were female $(86.4 \%), 11$ were male (13.6\%). Instructors' age ranged from 30-58 years old (mean 43.7 years). Seven females (77.8\%) and 2 males (22.2\%) participated.

Design: Participants received the prebriefing preparatory material in advance of their scheduled simulation via their didactic course folder. The researcher individually emailed the pre-briefing preparatory material to the simulation instructors, as this material was extensively detailed and intended solely for instructor use.

The RA emailed all participants an introductory email with links for study survey access via Survey Monkey. All participants (students and faculty) received pre-briefing preparatory material in advance. Instructors' preparatory material has additional information (Sample of Pre-briefing Preparatory Material; Table 1). In addition, all students received two links: Preparatory Material for SimulationStudent Survey (PMS-SS; Table 2) and LCJR/Scoring Sheet. Random sampling of participants received the link for the adapted Guide for Simulation Reflection (Table 3). Faculty also received an introductory email with a link to the Preparatory Material for Simulation-Instructor Survey (PMS-IS; Table 4).
Table 2: Preparatory Material for Simulation- Student Survey (PMS-SS)

Please check what course you are presently in

$\begin{array}{lrrrr}\text { N310 } & 15.4 \% & & \mathrm{~N} 312 & 26.0 \% \\ 31.2 \% & \mathrm{~N} 410 & 27.4 \% & & \mathrm{~N} 412\end{array}$

Demographic Information:

Male $11(13.6 \%) \quad$ Female $70(86.4 \%)$

Age 19-52/mean 21.88

Instructions: Based on the preparatory material you received for your simulation experience, please tick the appropriate box. Thank you.

1. Did you read the preparatory material prior to the simulation experience?

$$
\text { Yes } 100 \% \quad \text { No } 0
$$

If you answered no, please explain:

2. Did you feel you were prepared for the simulation?

$\begin{array}{lc}\text { Very prepared } & 21.0 \% \\ \text { Somewhat prepared } & 70.4 \% \\ \text { Slightly prepared } & 8.6 \% \\ \text { Not prepared } & 0\end{array}$

Please comment:

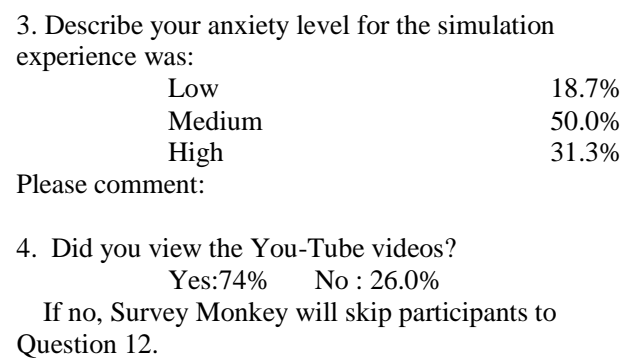

6. After reading the preparatory material, did you feel that the description of the "Roles during Simulation" prepared you for an understanding of what your role might be?

$\begin{array}{lr}\text { Very prepared } & 23.0 \% \\ \text { Somewhat prepared } & 55.4 \% \\ \text { Slightly prepared } & 18.9 \% \\ \text { Not prepared } & 2.7 \%\end{array}$


Please comment:

7. After reading the entire simulation scenario (including the additional medical history), did you feel you had enough information to effectively participate in the care of this patient?

$$
\text { Yes: } 89.2 \% \quad \text { No: } 10.8 \%
$$

If yes, how helpful was the entire simulation scenario description?

$\begin{array}{lc}\text { Very helpful } & 44.8 \% \\ \text { Somewhat helpful } & 45.7 \% \\ \text { Slightly helpful } & 8.1 \% \\ \text { Not helpful } & 1.4 \%\end{array}$

Please comment:

b) If no, please explain what additional information would have been helpful?

8. Overall, did the preparatory material provide you with a grasp of the simulation experience (Noticing)?

Please comment:

$$
\begin{array}{ll}
\text { Yes: } 96.2 \% & \text { No: } 3.8 \%
\end{array}
$$

If no, please explain:

9. Overall, did the preparatory material provide you with sufficient understanding of the simulation situation to respond effectively (Interpreting)?

Please comment:

$$
\text { Yes: } 91.3 \% \quad \text { No: } 8.8 \%
$$

If no, please explain:

10. Overall, did the preparatory material provide you with the information needed to effectively decide on a course of nursing action to participate in the care of this patient (Responding)?

$$
\text { Yes: } 87.5 \% \quad \text { No: } 12.5 \%
$$

Please comment:

If no, please explain:

11. Based on preparatory material and your experience during the simulation, please check all that apply to the how you felt after the experience (Reflecting): I felt:
a) more confident
$11.1 \%$
b) better prepared for future nursing care
c) my overall skills improved $\quad 9.9 \%$
d) I reflected on my own actions $\quad 18.5 \%$
e) I learned from my errors and colleagues $\quad 45.7 \%$

12. If you answered that you did not review the preparatory material prior to your simulation experience, did you feel that you were less prepared than students who had read the preparatory material?

Yes: $33.3 \%$ No $38.1 \% \quad$ Not sure 28.6\%*

Please comment:

13. If you have any additional comments, please provide them here:

Thank you.

*Not all percentages add up to 100 as all questions were not answered by all participants.
Table 3: Guide for Simulation Reflection using Tanner's (2006) Clinical Judgment Model (Adapted from Nielsen, Stragnell\&

Jester, 2007)

Instructions: This Adapted Guide for Simulation

Reflection is intended to help you think about your simulation experience and your nursing response to that experience. The specific situation you choose to reflect upon can the physiological patient problem (altered vital signs); description of your role during the simulation or with the other 'roles' during the simulation. The reflection may describe an ethical issue you encountered. Use this guide as a way to help you tell the story of the simulation situation you experienced.

The guide provides you with a way of thinking about care that supports the development of your clinical judgment. Although there are many ways of organizing your thinking about patient care and professional nursing practice, Tanner's (2006) Clinical Judgment Model provides the framework for the questions in this study guide.

Please check what course you are presently in:

N310 100\% (2) N312 100\% (2)

N410 100\% (2)

N412 $100 \%(2)$

Demographic Information:

Male $3(33.3 \%) \quad$ Female $5(66.7 \%)$

Introduction: Describe your simulation situation: Background:

- Consider experiences you have had that helped you provide nursing care in this

simulation situation. Describe your formal knowledge (e.g.: physiology, psychology, communication skills), previous nursing experience with a similar problem, and/or personal experiences that helped guide you as you worked with this simulated patient.

- Describe your beliefs about your role as the nurse working in this simulation situation.

- Describe any emotions you had about this simulation situation.

Noticing:

- What did you notice about the simulation situation initially?

- Describe what you noticed as you spent more time with the simulation patient/situation.

Interpreting:

- Describe what you thought about the simulation situation.

- Describe any similar situations you have encountered in clinical practice before. Describe any similarities and differences you observed when compared with this simulation situation.

- What other information (e.g.: assessment data, evidence) did you decide you needed as you considered the simulated situation? How did you obtain this additional information?

- What help with problem solving did you get from the preparation sheets? 


\section{DOI: $10.16899 /$ ctd.49922}

Your Conclusion: What did your observation and data interpretation lead to you believe?

How did they support your response to the simulated situation?

\section{Responding:}

- After considering the simulation situation, what was your goal for this simulated patient,

family and/or staff?

- What was your nursing response, or what interventions did you do? List your actions.

- Describe stresses you experienced as you responded to this simulated patient or others involved in the simulation.

Reflection-In-Action:

- What happened?

- How did the simulated patient, family, and/or staff respond?

- What did you do next?

Reflection-On-Action and Clinical Learning:

- Describe three ways your nursing care skills expanded during this simulation experience.

- Name three things you might do differently if you encounter this kind of situation again in the clinical setting.

- What additional knowledge, information, and skills do you need when encountering this kind of simulated situation or a similar situation in the future?

- Describe any changes in your values or feelings as a result of this simulated experience.

\section{Table 4: Preparatory Material for} Simulation- Instructor Survey (PMS-IS)

\author{
Please check what course you are presently in:

$\begin{array}{llll}\mathrm{N} 310 & 28.6 \% & \mathrm{~N} 312 & 28.6 \% \\ \mathrm{~N} 410 & 14.3 \% & \mathrm{~N} 412 & 28.6 \%\end{array}$ \\ Demographic Information: \\ Male $2(22.2 \%) \quad$ Female $7(77.8 \%)$
}

Age $30-58$, mean 43.7

Instructions: Based on the preparatory material you received for your simulation experience, please tick the appropriate box. Thank you.

1. Did you read the preparatory material prior to the simulation experience?

$$
\text { Yes: } 100 \% \quad \text { No: } 0
$$

If you answered no, please explain:

2. Did you feel you were prepared for the simulation?

$\begin{array}{ll}\text { Very prepared } & 66.7 \% \\ \text { Somewhat prepared } & 33.3 \% \\ \text { Slightly prepared } & 0 \\ \text { Not prepared } & 0\end{array}$

Please comment:

3. Describe your anxiety level for the simulation experience was:

$\begin{array}{ll}\text { Low } & 77.8 \% \\ \text { Medium } & 22.2 \% \\ \text { High } & 0\end{array}$

Please comment:

4. After reading the entire simulation scenario (including the additional medical history; simulation and scenario objectives), did you feel you had enough information to effectively participate in facilitating this simulation experience?

$$
\text { Yes: } 100 \% \quad \text { No: } 0
$$

If yes, how helpful was the entire simulation scenario description?

$\begin{array}{ll}\text { Very helpful } & 44.4 \% \\ \text { Somewhat helpful } & 55.6 \% \\ \text { Slightly helpful } & 0 \\ \text { Not helpful } & 0\end{array}$

If no, please explain what additional information would have been helpful?

5. After reading the preparatory material, did you feel that the description of the "Time Frame for Scenario" provided necessary information that enabled you to effectively teach?

$$
\text { Yes: } 100 \% \quad \text { No: } 0
$$

If yes, how helpful was the time frame for scenario?

$\begin{array}{ll}\text { Very prepared } & 55.6 \% \\ \text { Slightly prepared } & 44.4 \% \\ \text { Slighat prepared } & 0\end{array}$

Not prepared

If no, please explain what additional information would have been helpful?

6. After reading the preparatory material, did you feel that the "Debriefing and Guided Reflection" content provided you with enough information to assist in your facilitating of this simulation scenario?

$$
\text { Yes: } 100 \% \quad \text { No: } 0
$$

If yes, how helpful was the debriefing and guided reflection content?

$\begin{array}{ll}\text { Very helpful } & 66.7 \% \\ \text { Somewhat helpful } & 22.2 \% \\ \text { Slightly helpful } & 11.1 \% \\ \text { Not helpful } & 0\end{array}$

If no, please explain what additional information would have been helpful?

7. After reading the preparatory material, did you feel that the pathophysiology and additional material provided you with enough information to assist in your facilitating of this simulation scenario?

$$
\text { Yes: } 100 \% \quad \text { No: } 0
$$

If yes, how helpful was the pathophysiology and additional material?

$\begin{array}{ll}\text { Very helpful } & 44.0 \% \\ \text { Somewhat helpful } & 55.6 \% \\ \text { Slightly helpful } & 0 \\ \text { Not helpful } & 0\end{array}$

If no, please explain what additional information would have been helpful? 
DOI: $10.16899 /$ ctd.49922

$$
\begin{aligned}
& \text { 8. Did you view the YouTube videos assigned to the } \\
& \text { Yes: } 55.6 \% \quad \text { No: } 44.4 \%
\end{aligned}
$$

9. Overall, did the preparatory material provide you a grasp of the simulation experience (Noticing)? Yes: $100 \% \quad$ No:0

Please comment:

10. Overall, did the preparatory material provide you with sufficient understanding of the simulation situation to respond effective (Interpreting)? Yes: $88.9 \% \quad$ No: $11.1 \%$

Please comment:

11. Overall, did the preparatory material provide you with the information needed to effectively decide on a course of nursing action to participate in the care of this patient (Responding)? Yes: $88.9 \% \quad$ No: $11.1 \%$

Please comment:

12. Based on preparatory material and your experience during the simulation, please check all that apply to the how you felt after the experience (Reflecting): I felt:

a) more confident in guiding the simulation experience $25.0 \%$

b) better prepared to facilitate the simulation experience $62.5 \%$

c) my overall teaching skills improved 0

d) I reflected on my own actions

13. If you answered that you did not review the preparatory material prior to your simulation experience, did you feel that you were less prepared? Yes: $0 \quad$ No: $0 \quad$ Not sure :0

Please comment:

14. If you have any additional comments, please provide them here:

Thank you.

Pilot testing of seven students and four instructors were surveyed using these instruments to validate effectiveness [25]. Results demonstrated that the surveys were feasible and reliable with no revisions required though participants were unable to 'click all that apply' on one of the surveys. This limitation was rectified for this study.
Instruments: To maintain consistency with the theoretical framework, all surveys incorporated aspects of Tanner's Clinical Judgment Model. Consistency in language that was understood by both students and faculty helped establish rigor and validity to the instruments and study. The PMS-SS and PMSIS each contained 13 questions and explored how wellthe preparatory material prepared students and instructors for the simulation experience.LCJR/Scoring Sheet, had six questions, and explored students' responses to the simulation itself. Permission was received to utilize this survey. The Guide for Simulation Reflection, a nine question qualitative survey, was adapted to explore the reflective process during simulation. Both students and faculty decided which simulation experience they would complete the surveys on.

Data Analysis: Data from Survey Monkey was converted into an SPSS database. Levene's test for equality of variances was used to test the difference between the means of several subgroups of a variable. The regular Levene's test available through the one-way ANOVA procedure was utilized. Student comments were analyzed, along with quantitative results, to inform improvement of the simulation experience. Qualitative data analysis from the random sampling of one student for the Guide for Simulation Reflection was analyzed according to themes. A coding scheme was developed that included key concepts based on literature review and theory. During data analysis, new codes were developed for concepts and themes that emerged from the investigator's content analysis of the transcripts. Inter-rater reliability was maintained by working with a senior nurse researcher.

Limitations: The researcher recognizes that students and instructors self-select to read preparatory materials in advance and may not answer all survey questions, thus these may be 
viewed as a constraint. There were several student comments regarding the LCJRScoring Sheet. Though the students received a link with full explanations of the rubric scoring sheet definitions, many commented that they did not understand the rubric itself. The sample size may be considered too small for generalizability to other educational programs.

\section{RESULTS}

Overall, pre-briefing preparatory material for both students and instructors provided essential and adequate information for an effective and enhanced simulation learning experience.Students felt their ability to grasp the experience; understand the simulation situation; provide effective nursing actions; and reflect upon their experience was enhanced with the utilization of the pre-briefing preparatory material. Instructors felt more confident and prepared after reading the preparatory material. In addition, students' felt their clinical reasoning improved as they gained more selfconfidence and engaged in reflection on action.

\section{Student Results:}

All participants reviewed the pre-briefing preparatory material and the majority of students responded that after reading the preparatory simulation material, they felt they had enough information to effectively participate in the care of the simulated patient. As one student commented "The case was pretty straight forward; appropriate for our level of study. Based on the information provided, I was able to make a short list of assessments" while another student stated "The description contained a lot of information, and whatever was missing could have been asked of the patient during the experience, like in the real work because I'm assuming that you will not always have all of the information".

Students expressed that the pre-briefing material prepared them for the simulation.They sensed that they not only learned from their own errors and that of their colleagues but upon further reflection, they gained a deeper understanding and appreciation of their own actions and behaviors. This relates to their enhanced confidence with their nursing skills and ability to participate in the healing-caring process of others.Anxiety remains a concern for the majority of participants however, even withthe inclusion of preparatory material.One student stated, "My anxiety level was directly influenced by my level of preparedness. Had I been more completely prepared, my anxiety level would have been low and had I not been prepared at all, it would have been high". An overall theme that emerged regarding anxiety was being fearful of not knowing what to do. As one student stated "Although I was well aware that this was a learning experience and we were not being judged I still was fearful that I would come across as looking like a complete idiot".

Therapeutic communication was expressed as a necessary tool that still requires development. "Communication between the team members is important and how you communicated with the patient. If you're not communicating with the patient, you're going to miss something". Also, students realized the need to understand and perform appropriate nursing assessments. One student stated, "I need to improve my physical assessment skills, and my confidence with mediation calculation, administration and follow-up assessment". Simulation provides an invaluable format for enhancing and promoting therapeutic communication skills [22].

The inclusion of innovative teachinglearning strategies, such as YouTube and educational CDs, was viewed as very helpful. One student stated "It gave more info on the disorder and pathophysiology and showed how to conduct oneself in a simulation setting" while another student stated "After watching all of the 
YouTube videos', I felt I had a decent idea of how the simulation would go". YouTube can play a valuable role in teaching the communication and collaborative skills that future healthcare providers will require [23]. These teaching tools provide additional resources for students to gather more information. However, students decide on their own if they wish to utilize them, as they were not mandatory. Optimal use of YouTube and educational CDs requires an educator who is knowledgeable regarding the selection of these materials and skilled in incorporating them into the course content.

Overall, students felt their clinical judgment was enhanced, as they were able to prioritize medical data leading to improved patient care. The simulation experience promoted a calmer, more confident manner as a healthcare professional. Students felt their communication ability was improved and they engaged in self-evaluation, which further increased their confidence. As one student stated "This experience is great preparation for actual nursing experience. I always learn a tremendous amount of critical thinking skills from the simulation and from the interactions with my colleagues".

The Guide for Simulation Reflection validated these findings. The major themes that emerged from this adapted qualitative instrument yielded an overall improvement in students' perceived critical thinking skills as well as problem-solving. They felt more prepared for what they would anticipate in the workplace environment. Simulation is an excellent opportunity for learning patientcentered care (a primary goal of simulation for nursing students) while providing them with opportunities for knowledge application. As simulation continues to be implemented in healthcare education, the application of knowledge will need to be further explored. Areas that continue to need improvement are communication; appropriate nursing assessment skills and more confidence. All study survey findings correlate with pilot findings.

\section{Instructor Results:}

All instructors reviewed the pre-briefing preparatory material and felt that they were more prepared after reading the preparatory material. All had a better grasp of the simulation experience (Noticing); had sufficient understanding of the simulation situation (Interpreting); had enough information to effectively decide on a course of nursing action (Responding). As for reflecting, the majority felt better prepared to facilitate the simulation experience. Even educators have a learning curve, as one instructor stated "My first simulation - a motivating anxiety related to lack of experience which eased as the simulation progressed". All instructors stated that the preparatory material was very helpful in providing enough information to effectively participate in facilitating the simulation and debriefing process.

\section{DISCUSSION}

Pre-briefing preparation of students prior to simulation can enhance clinical judgment and reflective process. Pre-briefing material does enhance simulation learning outcomes; thus, they are an effective tool to further empower students to be active participants in their learning experience. By providing essential information to instructors, students' abilities to develop critical reasoning and problem-solving skills can be further enhanced. Based on student comments, some suggestions for enhancing the pre-briefing preparatory material included stating the importance of patient education and providing more detailed descriptions of the various roles of students during simulation (such as medication nurse, note taker, primary nurse).Decreasing anxiety continues to be a 
concern. Providing preparatory material does not fully eliminate anxiety, nevertheless does lower it. Studies reported that students experienced an initial increase in anxiety in simulation but also reported anxiety decreased with repeated exposure to simulation [3, 24]. While the paradox of anxious feelings with simulation exists, awareness and learning through simulation can occur and continue to formulate with reflection.

As one student stated "Overall, I felt that these simulation experiences are very helpful because they give a lot of insight into what we will come in contact with in our careers. It is also great because of the immediate feedback and therefore, the awareness of the need to improve and the way to go about improving". Another student stated "After simulation, I felt as if my overall skills improved because I went to look up things that I wasn't sure of, I went to learn the drug that I got stuck on during simulation and vow to never make a mistake of ever forgetting the adverse effects, I reflected upon my own actions and I learnt from my errors and colleagues. It was definitely a learning experience". While another student stated, "Overall, my skills for my career are improved every time I participate in a simulation". Finally, one student stated, "Perhaps another form of instruction would be to throw in some unexpected event to help us on our critical thinking skills".

There is a clear link between simulation, clinical judgment and reflective practice. By continually integrating simulation into nursing and medical curricula, students will be more prepared for the healing-caring environment. Developing more complex simulation scenarios will further prepare students to gain confidence and assessment skills necessary for the continued needs of our growing population.All healthcare providers must engage in clinical reasoning and reflection to enhance and advance their judgment. By preparing both faculty and students for the simulation experience, simulation outcomes can be achieved and enhanced, eventually leading to improved patient care outcomes. Contemporary rationales for the requisite development and utilization of simulation experiences are based on escalating patient acuity and the need to prepare our future health care providers for complex patient care scenarios [25]. By providing experiences whereby students can challenge their assumptions, gain experiences in a safe environment, communicate effectively in interprofessional relationships, patient care will be enhanced leading to dramatically improved overall patient outcomes. As our future health care practitioners gain more confidence in their roles, as they learn to reflect on their abilities to effectively participate in complex patient care, the overall educational implications are abundant. Today's hospitalized patients present with more complex and co-morbidities requiring comprehensive medical and nursing care. Simulation can provide experiences to further stimulate students understanding of the multitude of medical situations and co-morbid conditions they will encounter in their practice [26]. As medical and nursing competencies continue to evolve, so must educational outcomes and experiences. Simulation provides a creative, safe-environment to refine, revise, enhance and expand on the knowledge, skills and attitudes of today's future healthcare providers.

The outcomes of this study lead to improvements in the educational material that is prepared for faculty and students; thus, the overall educational process (clinical judgment and reflective practice) was improved and enhanced. These results are applicable to other schools of nursing and health professional schools that utilize simulation. It is important that both students and instructors are comfortable with the inclusion of simulation as a teaching strategy. For optimal outcomes, 
providing pre-briefing preparatory material can enhance both clinical judgment and reflective process for our future health care practitioners. Based on the results of this study, the researcher is now exploring translation of simulation clinical judgment to clinical practice.

\section{Conclusions}

Simulation is an effective teaching strategy to further enhance the educational learning process of future and current health care providers. By preparing future health care practitioners for clinical experiences, their overall effectiveness and efficiency in providing immediate and accurate care will be obtainable. This study demonstrated that clinical reasoning and judgment is enhanced through the integration of simulation. Insightful reflections on their own learning process furtherimprovetheir overall conceptual understanding of the intricacies of medical care. There is a clear link between simulation, clinical judgment and reflective practice. By continually integrating simulation, students will be more prepared for real workplace situations. Developing more complex simulation scenarios will further prepare our students to gain the confidence and assessment skills necessary for the continued needs of our growing population.

It is incumbent of all healthcare educators to equip their students with the best evidence-based practice and experiences. Prebriefing preparatory material provides students with information necessary to allow them to fully engage during the simulation experience, while decreasing anxiety and promoting critical thinking, clinical reasoning, reflective practice and confidence. Providing instructors with prebriefing preparatory material enhances their overall ability to effectively teach at a higher caliber while promoting professionalism and accuracy in patient care.
The outcomes of this study lead to improvements in the educational material that is prepared for both faculty and students; thus, the overall educational process (clinical judgment and reflective practice) was improved and enhanced. These results are applicable to other schools of nursing and health professional schools that utilize simulation. It is important that both students and instructors are comfortable with the inclusion of simulation as a teaching strategy. For optimal outcomes, providing pre-briefing preparatory material can enhance both clinical judgment and reasoning while promoting reflective practice of our future health care practitioners. Based on the positive results of this study, the researcher is now completing a study that explores the translation of simulated patient care to actual bedside care.

\section{REFERENCES}

1. Parker B, Myrick F. A critical examination of highfidelity human patient simulation within the context of nursing pedagogy. NursEduc Today 2009;29(3):322329.

2. Brown D, Chronister C. The Effect of Simulation Learning on Critical Thinking and Self-confidence When Incorporated Into an Electrocardiogram Nursing Course.ClinSim in Nsg2009;5(1):e45-e52.

3. Gore T, Hunt C, ParkerF, Raines K. The Effects of Simulated Clinical Experiences on Anxiety: Nursing Students' Perspectives.ClinSim in Nsg 2011;7(5):e175e180.

4. Swenty C, Eggleston B. The Evaluation of Simulation in a Baccalaureate Nursing Program. ClinSim in Nsg2011;(5):e181-e187.

5. Sullivan-Mann J, Perron CA, Fellner AN. The effects of simulation on nursing students' critical thinking scores: A quantitative study. Newborn and Infant Nsg Reviews 2009;9(2):111-116.

6. Fero LJ, O’Donnell JM, Zullo TG, Dabbs AD, Kitutu J, Samosky JT, Hoffman L.A. Critical thinking skills in nursing students: comparison of simulation-based performance with metrics. $\mathrm{J}$ of AdvNsg 2010;66(10):2182-2193.

7. Tanner CA. Thinking like a nurse: A research-based model of clinical judgment in nursing. $\mathrm{J}$ of Nsg Ed 2006;45:204-211.

8. Lapkin S,Levett-Jones T,Bellchambers H, Fernandez R. Effectiveness of Patient Simulation Manikins in Teaching Clinical Reasoning Skills to Undergraduate Nursing Students: A Systematic Review. ClSim in Nsg 2010;6(6):e207-e222.

Nielsen A, Stragnell S, Jester P. Guide for reflection using the clinical judgment model. $J$ of NsgEd 2007;46(11):513-516.

9. Lasater K.Clinical judgment: The last frontier for evaluation. NursEduc in Pract2011;11(2):86-92. 


\section{DOI: $10.16899 /$ ctd. 49922}

10. Kardong-EdgrenSE, Starkweather AR, Ward LD. The integration of simulation into a clinical foundations of nursing course: student and faculty perspectives. Int $\mathrm{J}$ of NursEducScholarsh 2008;5(1) doi: 10.2202/1548923X.1603

11. Howard V, EnglertN, Kameg K, Perozzi K. Integration of Simulation Across the Undergraduate Curriculum: Student and Faculty Perspectives. ClinSim in Nsg 2011;7(1):e1-e10.

12. Cantrell M. The Importance of Debriefing in Clinical Simulations. ClinSim in Nsg 2008;4(2):e19-e23.

13. Jeffries P. A framework for designing, implementing, and evaluating simulations used as teaching strategies in nursing. Nsg Ed Persp 2005;26(2):96-103.

14. Foronda C, LiuS, Bauman, E. Evaluation of Simulation in Undergraduate Nurse Education: An Integrative Review. ClinSim in Nsg 2013;9(10):e409-e416.

15. Jeffries P. Simulation in nursing education. From conceptualization to evaluation. New York: National League for Nursing, 2007.

16. Kardong-EdgrenS, Willhaus J, BennettD, Haydenm J. Results of the National Council of State Boards of Nursing National Simulation Survey: Part II.ClinSim in Nsg 2012;8(4):e117-123.

17. Lasater K. Clinical judgment development: Using simulation to create an assessment rubric. J of Nsg Ed 2007;26(11):496-503

18. Ashcraft A, Opton L. Evaluation of the Lasater Clinical Judgment Rubric. ClinSim in Nsg 2009;5(3):e130.

19. Schon D. The reflective practitioner. New York, NY: Basic Books, Inc, 1991.

20. Lundberg KM. Promoting self-confidence in clinical nursing students. Nurse Educ 2008;33(2):86-89.

21. Sleeper JA, Thompson C. The use of hi fidelity simulation to enhance nursing students' therapeutic communication skills. IntJof Nsg EdScholarsh 2008;5(1):1-12.

22. Author. Integrating YouTube into Nursing Curriculum, OJIN: The Online $\mathbf{J}$ of Iss in Nsg 200;16(3). http://nursingworld.org/MainMenuCategories/ANAMark etplace/ANAPeriodicals/OJIN/TableofContents/Vol-162011/No3-Sept-2011/Articles-Previous-Topics/YouTubeand-Nursing-Curriculum.html.

23. Hoffmann RL, O'Donnell JM, Kim Y. The effects of human patient simulators on basic knowledge in critical care nursing with undergraduate senior baccalaureate nursing students. Sim in Healthcare 2007;2(2):110-114.

24. Author. Preliminary exploration of simulation preparation, clinical judgment and reflective process. $\mathbf{J}$ of Cont Med 2012;2(3):141-150.

25. Author. Simulation and learning to care for patients: Acute alcohol withdrawal. Jof Cont Med 2013;3(1):2227. 